\title{
Megadalton chromatin remodelers: Common principles for versatile functions
}

\author{
Anna Jungblut ${ }^{1}$, Karl-Peter Hopfner ${ }^{2}$ \& Sebastian Eustermann ${ }^{1}$ \\ ${ }^{1}$ European Molecular Biology Laboratory (EMBL), Structural and Computational Biology Unit, \\ Heidelberg, Germany \\ ${ }^{2}$ Gene Center, Faculty of Chemistry and Pharmacy, Ludwig-Maximilians-Universität München, \\ Munich, Germany
}

\begin{abstract}
ATP-dependent chromatin remodelers determine the arrangement and composition of nucleosomes across eukaryotic genomes. Understanding the mechanisms of these enigmatic, macromolecular machines promises to reveal core principles of genome regulation and maintenance. However, the complexity of remodelers and their chromosomal substrates poses a major challenge for modern structural biology. Here, we review the recent breakthrough which provided by cryo-electron microscopy the first high-resolution insights into all four families of remodelers. We highlight the emerging structural and mechanistic principles with a particular focus on multi-subunit SWI/SNF and INO80/SWR1 complexes. A conserved architecture comprising a motor, rotor, stator and grip suggests a unifying mechanism how stepwise DNA translocation enables large scale reconfigurations of nucleosomes. A molecular circuitry involving the nuclear actin containing module establishes a framework for understanding allosteric regulation. Remodelers arise as programable hubs that enable differential processing of genetic and epigenetic information in response to the physiological status of a cell.
\end{abstract}




\section{Introduction}

Understanding the dynamic landscape of chromatin is one of the most intriguing challenges in modern structural biology. More than four decades ago, electron microscopy in conjunction with meticulous biochemistry enabled the discovery of the nucleosome [1,2], which led eventually to its molecular structure determined by $x$-ray crystallography $[3,4]$. However, even in light of the progress in the postgenomic era, the three-dimensional organization of the chromatin landscape and its dynamic interplay with the DNA transcription, repair and replication machineries, remain still largely unexplored at a detailed structural level. Most prominently, ample genetic and biochemical evidence established ATPdependent chromatin remodelers as decisive factors in chromatin organization [5]. Remodelers actively determine the position and composition of nucleosomes around gene promoters and other hallmark regions of eukaryotic genomes $[6,7]$ (Figure 1), yet we are only beginning to understand the underlying mechanism by which these enigmatic macro-molecular machines shape epigenetic information across the chromatin landscape.

The defining feature of remodelers are ATP dependent Snf2-type motor domains (Figure 1A), which form a branch within superfamily 2 helicases and nucleic acid translocases and catalyze ATP-dependent translocation of double stranded DNA [8,9]. Sequence features and additional domains flanking the conserved motor domain include auto-regulatory elements and serve as a platform by which remodeler interact with substrates and assemble into large, multi-subunit complexes. Based on their sequence homology and domain composition, nucleosome remodelers have been identified from yeast to human and classified into four families: ISWI, CHD1, SWI/SNF and INO80/SWR1 [10] (Figure 1A). There are several other Snf2-type ATPases such as Fun30/SMARCAD1, ALC1, MOT1 or RAD54 that act on nucleosomal or non-nucleosomal substrates and do not fall into these four families [11].

A hallmark of the SWI/SNF and INO80/SWR1 family remodelers is their macromolecular complexity comprising often more than fifteen different subunits in a mega-Dalton arrangement. Since an individual motor domain can be - in principle - sufficient to mobilize nucleosomes in vitro [12], this complexity is quite astonishing, but appears to be the result of intricate, highly regulated reactions on the nucleosome: INO80/SWR1 remodelers exchanges histone variants $[13,14]$, while SWI/SNF remodelers eject the entire histone octamer from DNA [15]; both families can also slide nucleosomes, albeit in different directions with respect to linker DNA $[15,16]$; finally, INO80 can generate evenly spaced nucleosomal arrays and position +1 nucleosomes flanking nucleosome depleted regions $[6,17]$ (Figure 1). It is yet unclear whether these distinct activities are experimental context-dependent products of the same basic mechanism, or whether distinct conformational or functional states result 
in different biological outcomes. In higher eukaryotes, the situation is even more complex as multisubunit remodelers can assemble in a combinatorial manner; 20 genes encoding 11 subunits of the human BAF complex may result in 288 theoretically possible variants of this SWI/SNF remodeler [18], allowing cell type- and cell state-specific composition and activities, or selective loss or gain of function of specific subunits during cancer evolution $[19,20]$. In contrast, CHD1 and ISWI family members copurify typically only with up to two or three subunits. Nevertheless, in their native chromatin environment, remodelers are likely to engage in numerous, functionally important interactions, similar to the combinatorial logic of transcription factors [21] or readers of histone modifications [22].

\section{Cryo-EM structures of all four families of ATP-dependent chromatin remodelers}

Advances of cryo-EM in conjunction with careful biochemical optimization revealed recently highresolution insights into all four families of remodelers (Figure 2A). Structures of individual Snf2-type ATPase motors and single subunit remodelers in complex with mono-nucleosomes [23-27] provided important insights of how the ATPase motor binds to nucleosomal DNA, of its allosteric regulation through substrate interactions and the mechanism of a DNA translocation step (see [28] for a recent review). In this review, we focus primarily on the more complex multi-subunit SWI/SNF and INO80/SWR1 remodelers. Their inherent complexity provides the opportunity to identify the emerging principles by which step-wise DNA translocation leads to nucleosome remodeling and how this mechanism is allosterically regulated.

The first high-resolution structures of multi-subunit remodelers in complex with nucleosomes were determined for the INO80 complex [29,30]. Recombinant co-expression strategies and gentle purification yielded mono-disperse fungal and human INO80 core complexes comprising all evolutionary conserved subunits $[29,30]$. Reconstitution with mono-nucleosomes and subsequent vitrification for cryo-EM required neither cross-linking nor addition of nucleotides [29]. Notably, seven INO80 subunits contain an ATPase-fold (Figure 2B) and some of these co-purified with ADP, while other co-purified with ATP. During the past months, structures of the SWI/SNF family were reported including endogenous fungal SWI/SNF [31] and RSC [32-34] complexes, as well as recombinant human BAF complex [35]. Collectively, all these structures revealed intriguing structural and functional parallels between the INO80/SWR1 and SWI/SNF families.

\section{A common architecture for SWI/SNF and INO80/SWR1 remodelers}

Previous genetic and biochemical studies mapped the modular organization of multi-subunit remodelers $[36,37]$. Based on the recent structural and functional insights, we propose a common architecture for SWI/SNF and INO80/SWR1 remodeler families: (1) a well-ordered core enables 
remodeling of a nucleosome via motor, stator and grip elements, (2) a nuclear actin module establishes allosteric control of the Snf2-type motor domain via its helical HSA and postHSA domains, (3) auxiliary, species specific subunits provide an additional layer of regulation, but their structural role is currently still largely elusive (Figure $2 \mathrm{~A}, \mathrm{~B}$ ).

The core of the remodeler clasps the nucleosome in between its Snf2-type ATPase motor and a grip element [29]. Figure 2 and 3 show cryo-EM structures of fungal SWI/SNF [31] and INO80 [29] core complexes as representative examples. Intriguingly, these structures revealed two different binding orientations of the Snf2-type motor domain, albeit at related super-helical locations (SHLs): the SWI/SNF ATPase motor binds SHL-2 with additional DNA contacts of its N-terminal lobe (N-lobe) to SHL+6, while INO80 ATPase motor binds SHL-6 and shows secondary contacts via its N-lobe to SHL+2 (Figure 2A). This provokes the question whether the same remodeler complex can bind nucleosomes in alternative configurations. Interestingly, an individual SWI/SNF ATPase motor show such behavior at SHL+2 and SHL-6 [38], while the motor domain of fungal SWR1 complex binds nucleosomes in the $\mathrm{SHL}+2$ configuration of the SWI/SNF complex [39]. However, the binding mode observed in cryo-EM structures of SWI/SNF and INO80/SWR1 complexes are fully consistent with biochemical foot-printing [14] and explain the direction of nucleosome sliding: SWI/SNF remodelers recognize exit DNA and slide mono-nucleosomes towards the end of a DNA template [15], whereas INO80 recognizes entry DNA and positions mono-nucleosomes towards the center [16].

Despite these differences, the overall modes of nucleosome binding by multi-subunit remodelers have common features. Equivalent histone and DNA contacts are recognized by grip subunits three DNA helical turns away from the motor domain (Figure 2A,3). Structure based mutations of the fungal INO80 targeting DNA interactions of evolutionary conserved Actin related protein 5 (Arp5) and INO80 specific subunit 6 (les6) revealed the critical role of the grip for nucleosome remodeling [29]. Moreover, Arp5 recognizes the histone octamer through a long helical insertion denoted as a grappler, revealing for the first time how a remodeler recognizes the acidic patch [29] (Figure 3A). The acidic patch at the surface of $\mathrm{H} 2 \mathrm{~A}$ and $\mathrm{H} 2 \mathrm{~B}$ histones is a hallmark interaction site for a multitude of chromatin factors [29,32-35], yet its functional relevance for ATP-dependent chromatin remodeling was not appreciated until recently [40]. In case of INO80, the acidic patch interaction is not only required for nucleosome sliding, but adjacent histone interactions of the grappler confer specificity for H2A.Z recognition [29]. The latter provides now a structural framework to revisit the controversially discussed role of INO80 during H2A.Z histone exchange and histone H3 K56 acetylation at gene promoters and DNA damage sites [41]. The acidic patch and equivalent DNA contacts are established by grip subunits of SWI/SNF remodelers 
(Figure 3B). Notably, disease related mutations of the grip subunit SMARCB1 highlight the physiological relevance of acidic patch interactions for the human BAF complex [42].

The architectural and functional parallels between the two remodeler families extend even further. Tentacle-like protein segments involved in regulation of the ATPase motor recognize the second acidic patch opposite of the grip (Figure 3A). The INO80 subunit les2 is likely to regulate the ATPase motor through a "throttle" helix and contains a long N-terminal region that wraps along the histone octamer and recognizes the second acidic patch [29]. In case of the yeast SWI/SNF remodeler RSC, it has been shown that the Snf2-ATP coupling (SnAC) domain C-terminal of the Snf2-type ATPase motor protein regulates its activity and interacts directly with the acidic patch [32]. Chromatin factors are prone to bind nucleosomes, due to its inherent symmetry, as a dimer. Recognition of both gyres via acid patch contacts ensures monomeric binding of SWI/SNF and INO80/SWR1 remodelers and uni-directional translocation of DNA by a single ATPase motor. The binding orientation of SWI/SNF and INO80/SWR1 remodeler is most likely depend on recognition of extra-nucleosomal DNA, as discussed further below. Dimeric binding by remodelers has been reported $[43,44]$, but such a scenario requires asymmetric regulation of the two ATPase motors to prevent futile cycles of DNA translocation in opposing direction. Given the direct link to the ATPase motor, recognition of the acidic patch e.g. by les2 and the SnAC domains are prime candidates for such a regulation. Moreover, future studies may reveal whether some remodeling reactions, such as histone exchange and ejection, may even rely on two ATPase motors that are simultaneously active.

\section{A rotor, motor, stator and grip: how DNA translocation leads to nucleosome remodeling}

On their own, ATPase motors track along the minor groove of a double stranded DNA substrate, which led early on to the hypothesis that remodelers must hold on to one part of the nucleosome and keep the ATPase motor in place to alter DNA-histone interactions of another part $[9,45]$. The common architecture of INO80/SWR1 and SWI/SNF complexes provides now finally structural evidence for this principle.

Cryo-EM structures of SWI/SNF and INO80/SWR1 complexes show a well-ordered scaffold of core subunits which bridges the ATPase motor domain on one site of the nucleosome and the grip subunits on the other side, altogether generating a multi-valent interaction. In case of the INO80/SWR1 family, this scaffold is established by the hetero-hexameric AAA+ATPase Rvb1/2 that captures the long family specific insertion segment of the ATPase motor protein $[29,30,46]$ (Figure $2 A$ ). In case of the SWI/SNF family, a scaffold of subunits (also denoted as a body) assembles via the N-terminal pre- 
helical SANT associated (pre-HSA) domain of the motor protein, while its helical HSA domain connects it to the ATPase domain [31-35]. Like the design of an "electromotor", an analogy we adopted from the analysis of ATP synthetases [47], we proposed a stator-like function for the scaffold: the stator enables processive translocation of DNA, as it mechanically couples the ATPase motor to the stationary nucleosome interaction of the grip [29] (Figure 3C). Indeed, structure-based mutations of the grip led to futile cycles of ATP-hydrolysis in vitro [29] and abrogated chromatin remodeling by BAF complex in vivo [42]. Other DNA related processes such as promoter opening by the Snf2-type ATPase Ssl2, as part of the yeast RNA polymerase II pre-initiation complex [48], may rely on similar principles (Figure 3C).

Insights into the mechanism of a DNA translocation step have been now obtained from cryo-EM structures of individual ATPase motors in different nucleotide states $[49,50]$. The ratchet-like mechanism involves, as previously proposed [51], a helical twist defect of nucleosomal DNA [26,50]. A screw-like rotation of the DNA is driven by translocation of a "tracking strand" [9] and proceeds with a step size of 1-2 base pairs per hydrolyzed ATP molecule. Helical twist diffusion [51,52] might explain the residual nucleosome sliding activity of individual ATPase motors, even if stochastic DNA translocation events are frequently intermitted by dissociation. However, nucleosome sliding by remodelers occurs often in larger visible steps than 1-2 base pairs; step sizes of up to 10-20bp and corresponding paused intervals have been reported for INO80 [53,54]. Moreover, histone exchange or ejection reactions require extensive disruption of histone-DNA contacts and are thus likely to require successive rounds of stepwise DNA translocation by the ATPase motor domain to lead a detectable larger step size as probed by the particular assay. Such a mechanism, where several small hidden steps lead to a larger remodeling step is similar to the mechanism proposed for certain helicases unwinding DNA [55].

The ATPase motor, DNA rotor, scaffold stator and nucleosome interaction grip of SWI/SNF and INO80/SWR1 complexes provide now first insights into a unifying mechanism by which remodeler may conduct such large-scale reconfigurations of nucleosomes (Figure 3B). The multi-valent binding mode ensures not only persistent binding of the ATPase motor for successive rounds of stepwise DNA translocation, but it provides also the means to build up a DNA strain against the grip subunits [29]. In case of INO80, DNA translocation would result in a DNA strain between the ATPase motor at SHL-6 and the Arp5-les6 grip at SHL-2/-3 [29]. Persistent disruption of DNA H2A-H2B contacts in this region is consistent with biochemical probing and provides an elegant explanation for histone exchange $[29,53]$. Nucleosome sliding might occur when the DNA strain is sufficiently large for propagation 
beyond the grip [29,53], which in turn explains, similar to a DNA loop propagation model [52], the large step size of nucleosome sliding. Given the common architecture of multi-subunit remodelers, the same principles may apply for different remodeling reactions by SWR1 and SWI/SNF complexes. Notably, stepwise translocation of DNA by SWI/SNF ATPase motors at SHL-2 would generate a DNA strain against the grip at $\mathrm{SHL}+1 /+2$, which may explain histone octamer ejection by destabilizing DNA contacts of histone H3 (Figure 3B). Moreover, DNA translocation may also result in different types of strains, such as deformations of the histone octamer [24].

Future studies will build on the advances of time-resolved cryo-EM to visualize the molecular choreography of such events. Notably, DNA translocation via a screw-like forward rotation would lead to under- and over-winding of DNA "in-front" and "behind" the ATPase motor (Figure 3B).

Remodelers such as INO80 and RSC might thereby probe the mechanistic properties of promoter DNA sequences during nucleosome positioning [6] and ejection [56]. Moreover, long range allosteric communication via the conformational plasticity of DNA might have intriguing implications for the interplay of remodelers with other macro-molecular machines such as transcriptional complexes and topoisomerases.

\section{A molecular circuitry for allosteric regulation: the role of nuclear actin}

Nuclear actin ( $\mathrm{N}$-actin) and actin related proteins (Arps) are a common yet enigmatic feature of INO80/SWR1 and SWI/SNF remodelers. An evolutionary conserved pair of monomeric $\mathrm{N}$-actin and Arp4 forms the core of an N-actin/Arp module, which is present in all of these remodelers, except yeast SWI/SNF and RSC complexes, where Arp4 and N-actin are replaced by a structurally related pair of Arp7 and Arp9 [57] (Figure 2). The N-actin/Arp module plays a pivotal role in cellular differentiation, development and stress response, while mutations in corresponding genes, such as the human Arp4 orthologue BAF53, have been frequently linked to human diseases such as cancer $[58,59]$.

The recent progress in structural and biochemical studies of INO80/SWR1 and SWI/SNF complexes provide now insights into a conserved function of the $\mathrm{N}$-actin module as an allosteric regulator of chromatin remodeling. While in SWI/SNF complexes, the $\mathrm{N}$-actin module is more rigidly localized through interactions with the motor domain, high resolution analysis of the entire INO80 remodeler was limited by conformational heterogeneity of the $\mathrm{N}$-actin/Arp module in respect to the wellordered core, but an integrated approach combining cryoEM and $\mathrm{x}$-ray structures led to a composite $1 \mathrm{MDa}$ structural model $[29,60]$ (Figure 4A). In INO80 the N-actin/Arp module recognizes $40 \mathrm{bp}$ linker 
DNA at DNA entry site of the nucleosome core particle proximal to the Snf2-type ATPase motor, consistent with mapping of INO80 subunits in vitro [61] and in vivo [62]. The 120A long HSA domain of the Snf2-type motor protein plays a central role in this organization: (1) it assembles Arp4, N-actin and Arp8, (2) it directly recognizes DNA, and (3) it forms a continuous helical connection to the postHSA domain that establishes in turn a direct link to the ATPase motor. The recent cryo-EM structures of SWI/SNF remodelers show a different configuration of the N-actin module that accommodates its role as a bridging element between the ATPase motor and the stator scaffold, as illustrated by the RSC structure[32] in Figure 4B. The post-HSA domain remains bound to the N-lobe of ATPase motor, but its helical connection to the HSA domain is interrupted. The HSA domain packs on top of the ATPase motor and adopts a reverse orientation in comparison to INO80. Nevertheless, due to the binding mode of the ATPase motor at SHL-2, the N-actin module points still towards the linker DNA at the exit site of the nucleosome, where other SWI/SNF subunits, such as Rsc2, Rsc3 and Rsc30 of the yeast RSC complex[32] or ARID1A/B of human BAF complexes[63], have been implicated in DNA binding.

The postHSA domain and its interaction with the protrusion I element of the ATPase motor is a highly conserved features of INO80/SWR1 and SWI/SNF remodelers [60] (Figure 4C). Genetic and biochemical evidence suggested a regulatory role of these features in coupling ATP-hydrolysis to DNA translocation [15]. The protrusion I element of the $\mathrm{N}$-lobe is connected via a brace helix to the protrusion II element of the C-lobe; recent cryo-EM structures show a concerted interplay of these elements during DNA translocation $[29,38,50]$. Based on the structural and mechanistic insights into the INO80 complex, we proposed a model for its allosteric regulation: DNA sensing by the HSA domain promotes, via the configuration of the post-HSA domain, coupling of ATPase motor [60]. Such coupling is required to build up a DNA strain for nucleosome remodeling, and to prevent backslippage of the DNA (Figure 4D). Indeed, structure based mutations targeting the DNA interaction of the HSA domain [60] or a limiting length of linker DNA [54] abrogated nucleosome sliding and uncouple it from futile cycles of ATP-hydrolysis. Sensing linker DNA is a hallmark of chromatin remodelers and may provide the mechano-chemical means to conduct higher order remodeling reactions. A molecular ruler mechanism for adjusting the spacing between nucleosomes has been proposed for the ISWI family [64]. Future studies will build on the insights described here to understand how multi-subunit remodelers conduct such activities.

Similar principles may apply for SWI/SNF remodelers. It has been shown that the assembly of the Nactin module is dependent on auxiliary factors and the nucleotide state of yeast Arp7 and Arp9 [65- 
67]. A fully assembled $\mathrm{N}$-actin module might not only promote the coupling of the ATPase motor, but stabilizes also the helical conformation of the HSA domain, which is in turn required for the stator-grip function of the remodeler. Since ejection of a histone octamer must rely on extensive remodeling of histone-DNA contacts, it likely depends on an efficient coupling of ATP-hydrolysis to DNA translocation as well as on a rigid connection to the stator-grip element. This synergy may also explain the different remodeling outcomes, as observed for the RSC complex [15], and its allosteric regulation. Intriguingly, the human Arp4-orthologue BAF53 exists in different isoforms, which play a key role in neuronal development [68]. It will be intriguing to ask whether such subunits change the coupling efficiency of the BAF complex. The unifying core principles of remodeling may thus suggest a mechanism by which combinatorial composition of multi-subunit remodelers might play a critical role for allosteric regulation and fine tuning of their activities.

\section{Concluding remarks}

In the post-genomic era, DNA sequencing-based mapping and light microscopy revealed unprecedented insights into the spatio-temporal organization of the chromatin landscape. However, understanding how the cellular machinery processes genetic and epigenetic information in response to changes in its environment, requires a comprehensive and detailed picture (or ideally movies) of how chromatin factors communicate with each other in a context dependent manner.

Cryo-EM structures provide now finally a first glimpse into the complexity ATP-dependent chromatin remodeler, as reviewed here for members of the INO80/SWR1 and SWI/SNF families. While these megadalton remodelers show even in context of "simple" mono-nucleosomes a substantial structural and functional diversity, we propose a set of common mechanistic principles including a motor-statorgrip based architecture and allosteric regulation of the motor by the $\mathrm{N}$-actin containing module. Based on these insights, we predict mechanistic cycles with profound structural dynamics and intersubunit allostery that may thus provide ample of opportunity for an additional layer of selective regulation e.g. by epigenetic marks or pioneering factors. In their native chromatin environment, remodelers are not solely "molecular stir bars" that randomly mobilize nucleosomes, but they may act as programable hubs that regulate gene expression and maintenance by responding to a diverse set of signals with specific remodeling reactions.

Challenges in future studies are therefore to visualize remodelers on more complex, physiologically relevant chromatin substrates including for instance the transcriptional machinery and to trap critical functional intermediates or perform time-resolved studies. In addition, while we have now revealed 
the "biochemical" function of widely shared subunits (such as N-actin and the Rvb1/2 chaperone remodelers) acting as allosteric regulator and stator scaffold modules, the "biological" function of having or retaining these subunits merits further investigation. For instance, diversification of actinArp4 into Arp9-Arp7 in yeast RSC shows that, in principle, actin at the HSA domain can evolve into a paralog, adopting its structural role. This paralog can evolve much easier than actin, which could arguably be regarded as an advantage for the remodeler to adapt to changing requirements. The fact that $\mathrm{N}$-actin is evolutionary retained in mammalian and fungal remodelers of the SWI/SNF and INO80 family suggests that $\mathrm{N}$-actin does not merely play an architectural building block role, but has perhaps a more global biological function underneath. Similar arguments can be made for the Rvb1/2 chaperone. The breakthrough structures achieved in the past 3 years provide now a framework to address the biology of chromatin remodeling on the basis of structural molecular mechanisms. 
A

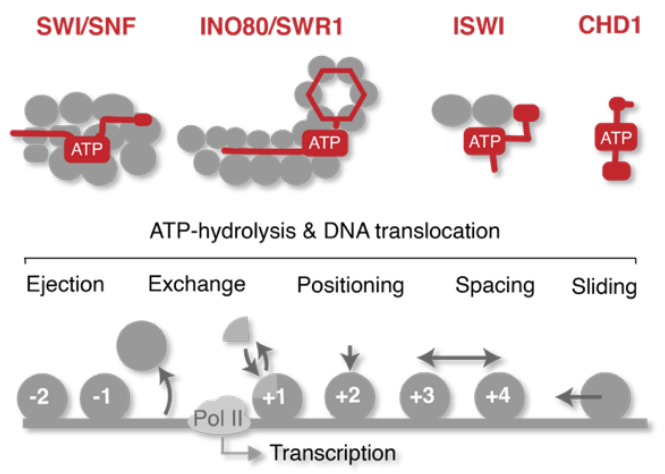

B

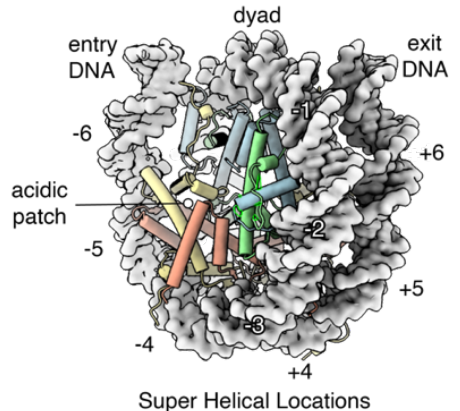

\section{Figure 1}

A. Four families of remodelers shape the chromatin landscape. Snf2-type ATPase motor proteins (red) are the defining feature of each remodeler family and serve as an assembly platform. A common mechanism of ATP-dependent DNA translocation underlies the different remodeling reactions [5]. Remodelers generate hallmark features of chromatin around gene promoter regions [6]. A. Structure of the nucleosome core particle. Positive and negative super-helical locations are assigned according to the direction of DNA translocation. The $\mathrm{H} 2 \mathrm{~A}-\mathrm{H} 2 \mathrm{~B}$ acidic patch of the histone octamer ( $\mathrm{H} 2 \mathrm{~A}$ yellow, $\mathrm{H} 2 \mathrm{~B}$ red, $\mathrm{H} 3$ blue, $\mathrm{H} 4$ green) is conserved interaction site for diverse chromatin factors. 
A
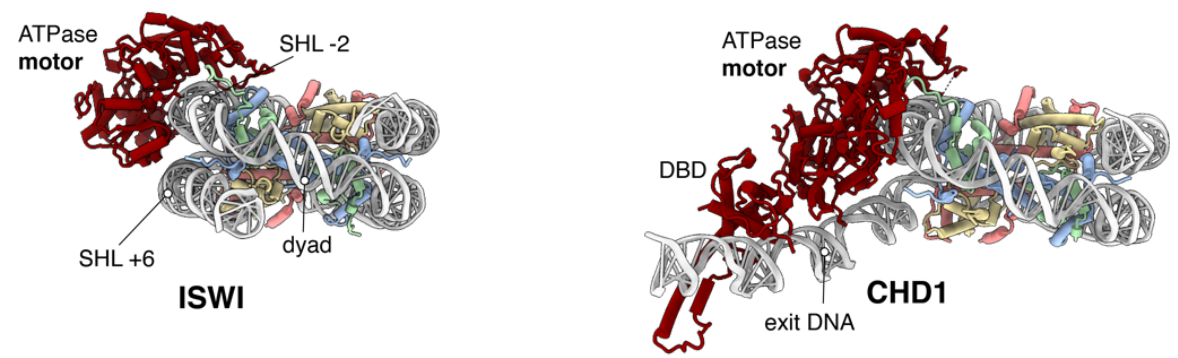

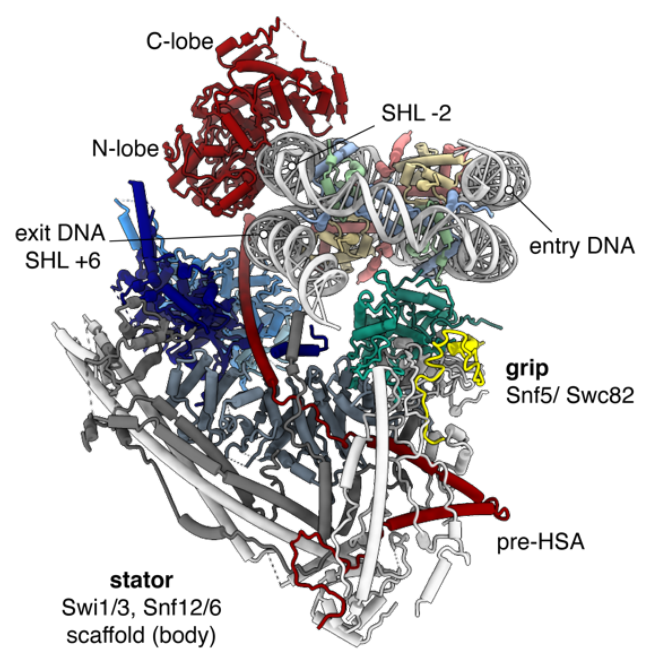

SWI/SNF

B

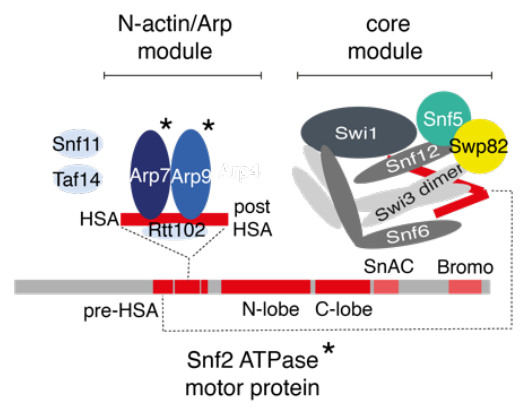

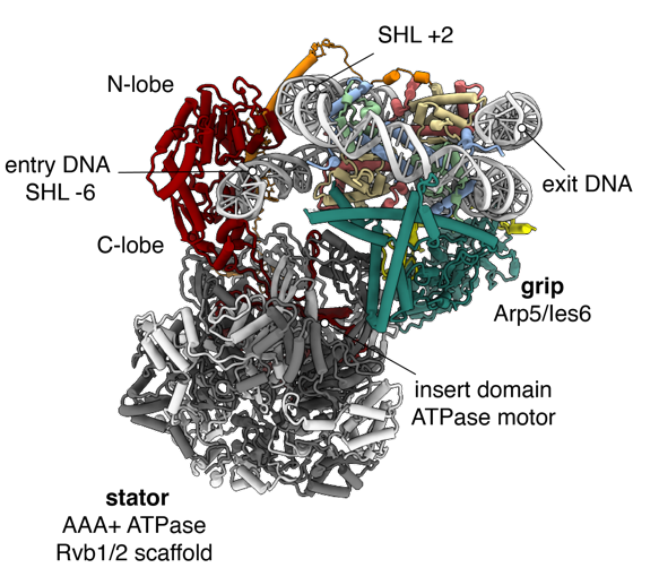

INO80

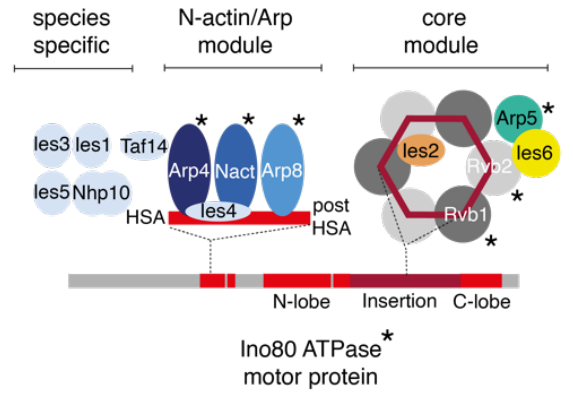

\section{Figure 2}

A. Structural comparison of the four chromatin remodeler families. The eponymous, fungal representatives of each family are shown: ISW1 [49], Chd1 [69], SWI/SNF complex[31] and INO80 complex[29]. SHL interaction sites of Snf2-type ATPase motor (red) as well as entry and exit DNA are indicated on the nucleosome core particle (NCP). Histones are color coded according Figure 1. Core subunits assembled into a stator scaffold (gray, also denoted as a body complex) via Snf2 pre-HSA domain and Ino80 insertion domain, respectively. NCP recognition subunits are color coded in green (Arp5,Snf5) and yellow (les6,Swc82).The N-actin/Arp module, included in the SWI/SNF structure, is colored in blue hues and binds a helical HSA domain of Snf2 (also in red). B Common architecture of the SWI/SNF and INO80 remodelers comprising the core, the $\mathrm{N}$-actin/Arp and a species-specific module. The assembly of remodeler subunits of $\mathrm{S}$. cerevisiae SWI/SNF and INO80 onto the motor subunits Swi2/Snf2 and Ino80 are illustrated by red bars connected with dotted lines. Subunits of similar function or homology are colored likewise for INO80 and SWI/SNF (same color scheme as in A). 
A

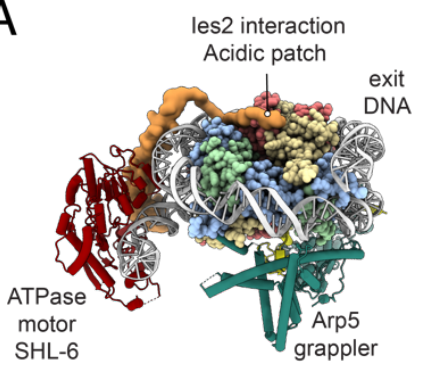

exit
DNA
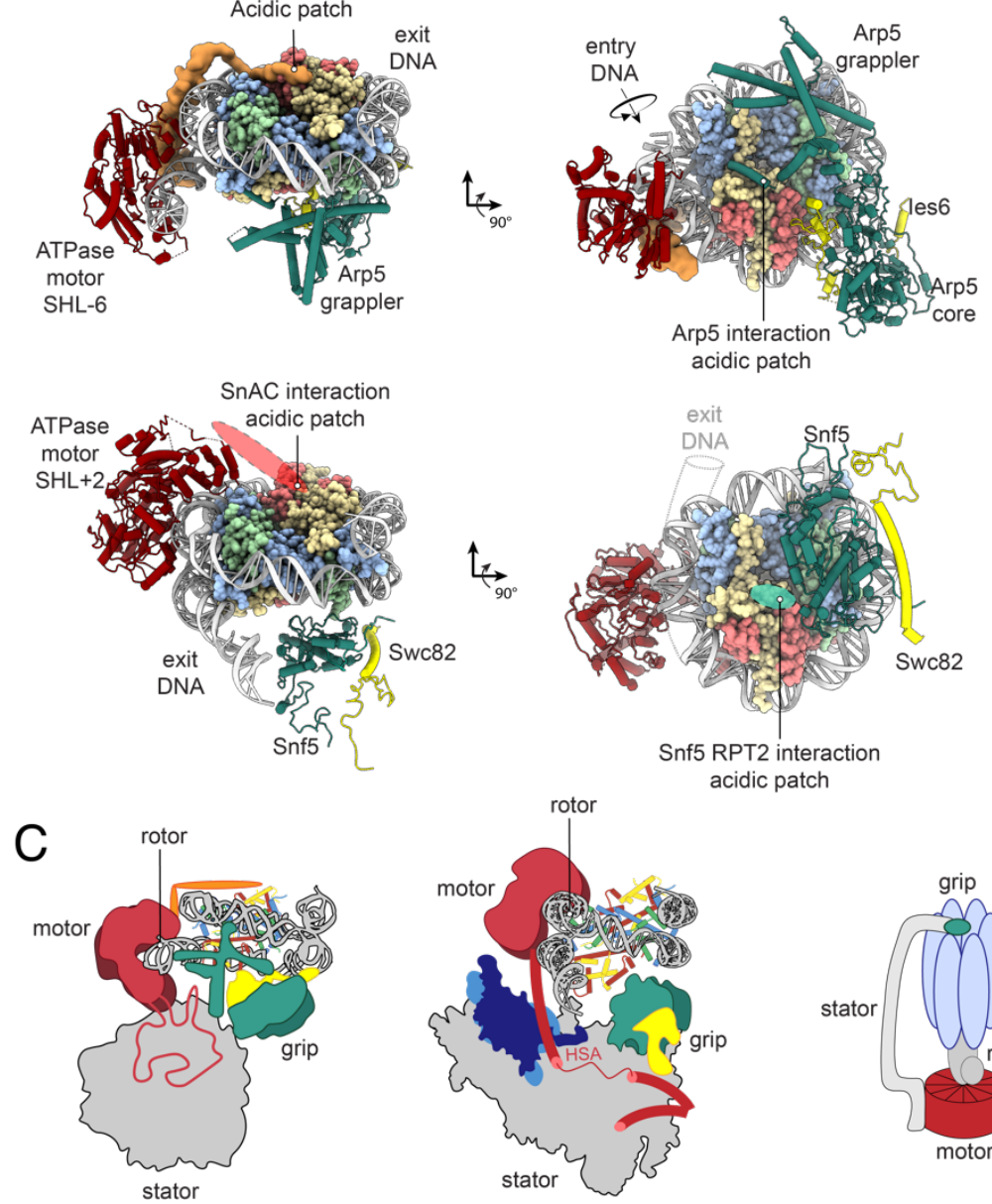

B

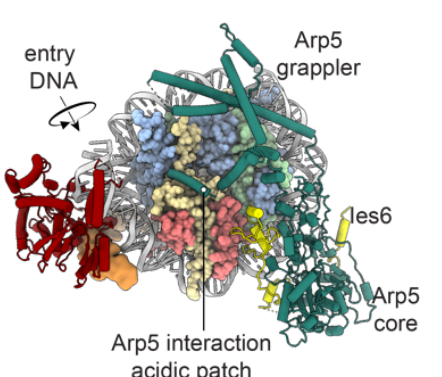
acidic patch

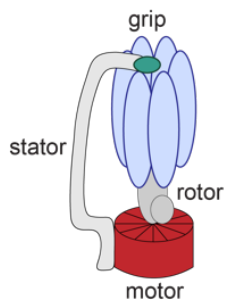

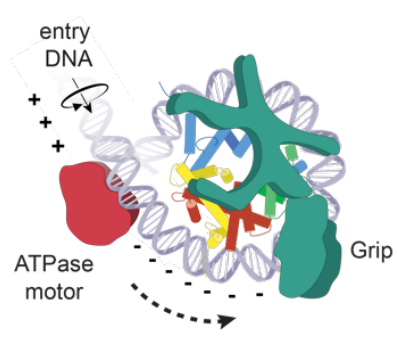

build up DNA strain

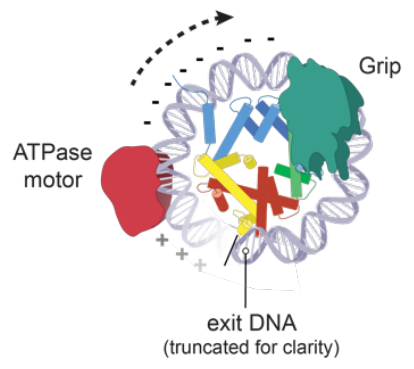

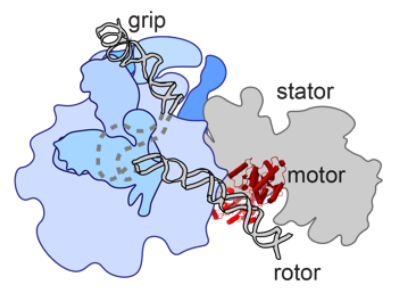

\section{Figure 3}

A. Conserved mode of multi-valent nucleosome recognition. Fungal INO80[29] (upper panel) and yeast SWI/SNF [31] (lower panel) subunits in direct contact with nucleosome are shown as representative examples (same color code as in Figure 2). The grip binds nucleosomal DNA about three helical turns away from the ATPase motor, while acidic patch contacts on each site of the histone octamer establish a sandwich like configuration. The interaction of the SWI/SNF SnAC domain is inferred from a homologous interaction observed for yeast RSC complex (ref). B. Common remodeling mechanism. Successive rounds of 1-2bp steps of DNA translocation build up a DNA strain against the grip. DNA underwinding and over-winding is indicated by minus and plus symbols, respectively. In case of INO80, exposure of $\mathrm{H} 2 \mathrm{~A}-\mathrm{H} 2 \mathrm{~B}$ may lead to histone exchange, while propagation beyond the grip leads to nucleosome sliding. In case of SWI/SNF, underwinding would build up DNA strain at $\mathrm{H} 3$ contacts, which may thus lead to histone ejection. D. Cellular machineries with "electromotor" design. Schematic side views of INO80, SWI/SNF, ATP synthetase and Polymerase II pre-initiation complex. Motor is colored in red, stator - in grey, grip - in green. In Pol II pre-initiation complex the Ss/2 helicase motor (red) of TFIIH is kept in place by the TFIIH stator (light gray). Translocation of DNA towards RNA Polymerase II would lead to DNA underwinding and facilitated promoter opening. Based on PDB: 5oqj and [48]. 
A

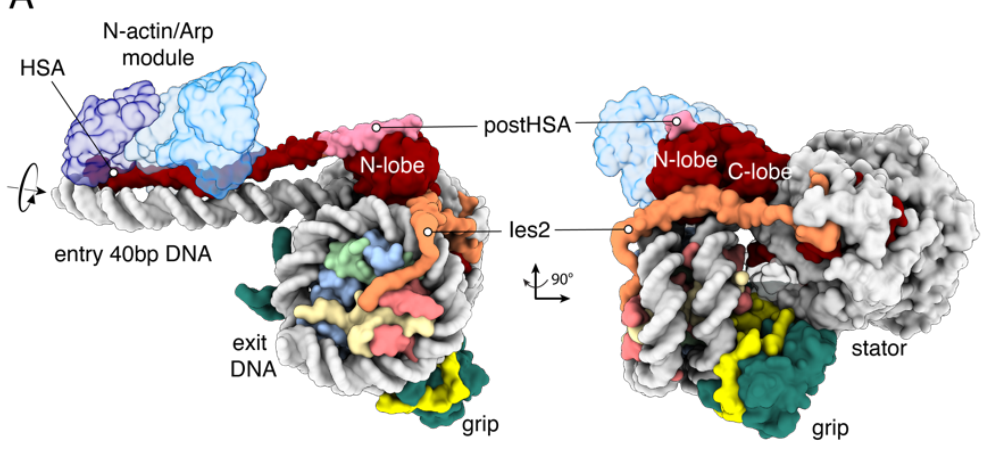

B

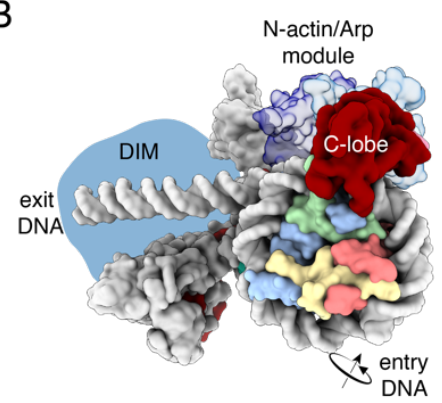

C
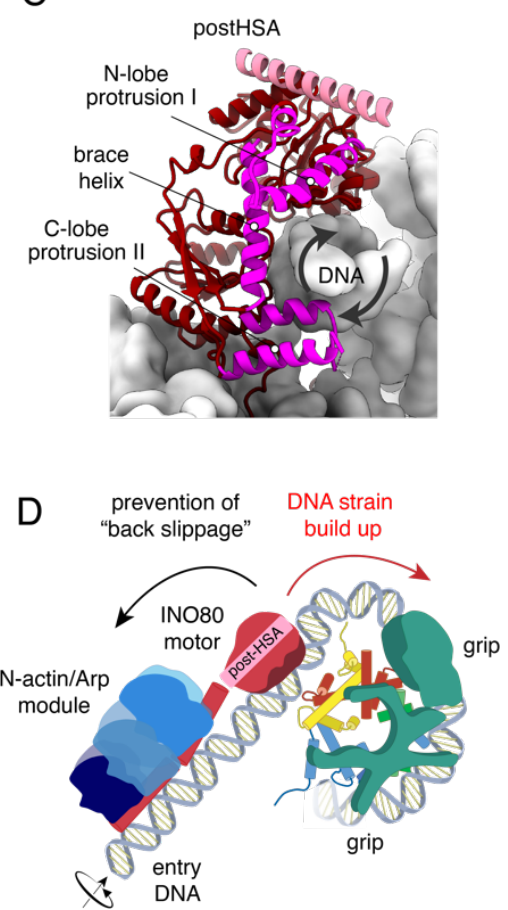

\section{Figure 4}

A. Composite cryoEM and x-ray structural model of INO80 core complex and N-actin/Arp module[29,60] recognizing 40bp linker DNA. HSA domain (red) assembles the N-actin/Arp module via barbed end interactions and connects it to the post-HSA domain (pink), which provides a direct link to the N-lobe of the ATPase motor. B. CryoEM structure RSC remodeler[32]. N-actin/Arp module bridge the ATPase motor (red) and the scaffold (light grey), with helical connection of the post-HSA domain to the HAS domain being interrupted. C. Snf2type ATPase motor of INO80. Protrusion I element of the N-lobe is connected to protrusion II of the C-lobe via a C-terminal brace element. All three elements are required for efficient coupling ATP-hydrolysis to DNA translocation (indicated by arrows) and their interplay is modulated by the binding of the postHSA domain (pink) D. Allosteric mechanism of linker DNA sensing by INO80. Recognition of linker DNA by HSA domain establishes allosteric communication with postHSA domain which in turn promotes coupling of ATP-hydrolysis and DNA translocation [60]. Sufficient build of DNA strain is required for DNA propagation beyond the grip and nucleosome sliding[29]. 


\section{Bibliography}

1. Kornberg RD, Thomas JO: Chromatin structure; oligomers of the histones. Science 1974, 184:865-868.

2. Olins AL, Olins DE: Spheroid chromatin units (v bodies). Science 1974, 183:330-332.

3. Richmond TJ, Finch JT, Rushton B, Rhodes D, Klug A: Structure of the nucleosome core particle at 7 A resolution. Nature 1984, 311:532-537.

4. Luger K, Mäder AW, Richmond RK, Sargent DF, Richmond TJ: Crystal structure of the nucleosome core particle at 2.8 A resolution. Nature 1997, 389:251-260.

5. Clapier CR, Iwasa J, Cairns BR, Peterson CL: Mechanisms of action and regulation of ATP-dependent chromatin-remodelling complexes. Nat Rev Mol Cell Biol 2017, 18:407-422.

6. Krietenstein N, Wal M, Watanabe S, Park B, Peterson CL, Pugh BF, Korber P: Genomic Nucleosome Organization Reconstituted with Pure Proteins. Cell 2016, 167:709-721.e712.

7. Zhang Z, Wippo CJ, Wal M, Ward E, Korber P, Pugh BF: A packing mechanism for nucleosome organization reconstituted across a eukaryotic genome. Science 2011, 332:977-980.

8. Saha A, Wittmeyer J, Cairns BR: Chromatin remodeling by RSC involves ATP-dependent DNA translocation. Genes Dev 2002, 16:2120-2134.

9. Durr H, Korner C, Muller M, Hickmann V, Hopfner KP: X-ray structures of the Sulfolobus solfataricus SWI2/SNF2 ATPase core and its complex with DNA. Cell 2005, 121:363-373.

10. Clapier CR, Iwasa J, Cairns BR, Peterson CL: Mechanisms of action and regulation of ATP-dependent chromatin-remodelling complexes. In Nature Publishing Group. Edited by: Nature Publishing Group; 2017:407-422. vol 18.]

11. Hopfner KP, Gerhold CB, Lakomek K, Wollmann P: Swi2/Snf2 remodelers: hybrid views on hybrid molecular machines. Curr Opin Struct Biol 2012, 22:225-233.

12. Liu X, Li M, Xia X, Li X, Chen Z: Mechanism of chromatin remodelling revealed by the Snf2-nucleosome structure. Nature 2017, 544:440-445.

13. Mizuguchi G, Shen X, Landry J, Wu WH, Sen S, Wu C: ATP-driven exchange of histone H2AZ variant catalyzed by SWR1 chromatin remodeling complex. Science 2004, 303:343-348.

14. Brahma S, Udugama MI, Kim J, Hada A, Bhardwaj SK, Hailu SG, Lee TH, Bartholomew B: INO80 exchanges H2A.Z for H2A by translocating on DNA proximal to histone dimers. In Nature Communications. Edited by; 2017. vol 8.]

15. Clapier CR, Kasten MM, Parnell TJ, Viswanathan R, Szerlong H, Sirinakis G, Zhang Y, Cairns BR: Regulation of DNA Translocation Efficiency within the Chromatin Remodeler RSC/Sth1 Potentiates Nucleosome Sliding and Ejection. In Molecular Cell. Edited by: Elsevier Inc.; 2016:453-461. vol 62.]

16. Udugama M, Sabri A, Bartholomew B: The INO80 ATP-dependent chromatin remodeling complex is a nucleosome spacing factor. Mol Cell Biol 2011, 31:662-673.

17. Oberbeckmann E, Niebauer V, Watanabe S, Farnung L, Moldt M, Schmid A, Cramer P, Peterson CL, Eustermann S, Hopfner K-P, et al.: Ruler elements in chromatin remodelers set nucleosome array spacing and phasing. 2020:2020.2002.2028.969618.

18. Wu JI, Lessard J, Crabtree GR: Understanding the words of chromatin regulation. Cell 2009, 136:200-206.

19. Schick S, Rendeiro AF, Runggatscher K, Ringler A, Boidol B, Hinkel M, Majek P, Vulliard L, Penz T, Parapatics $\mathrm{K}$, et al.: Systematic characterization of BAF mutations provides insights into intracomplex synthetic lethalities in human cancers. Nat Genet 2019, 51:1399-1410.

20. Kadoch C, Crabtree GR: Reversible disruption of mSWI/SNF (BAF) complexes by the SS18-SSX oncogenic fusion in synovial sarcoma. Cell 2013, 153:71-85.

21. Wolberger C: Combinatorial transcription factors. Curr Opin Genet Dev 1998, 8:552-559.

22. Ruthenburg AJ, Li H, Patel DJ, Allis CD: Multivalent engagement of chromatin modifications by linked binding modules. Nat Rev Mol Cell Biol 2007, 8:983-994.

23. Sundaramoorthy R, Hughes AL, El-Mkami H, Norman DG, Ferreira H, Owen-Hughes T: Structure of the chromatin remodelling enzyme Chd1 bound to a ubiquitinylated nucleosome. Elife 2018, 7.

24. Armache JP, Gamarra N, Johnson SL, Leonard JD, Wu S, Narlikar GJ, Cheng Y: Cryo-EM structures of remodeler-nucleosome intermediates suggest allosteric control through the nucleosome. Elife 2019, 8.

25. Liu X, Li M, Xia X, Li X, Chen Z: Mechanism of chromatin remodelling revealed by the Snf2-nucleosome structure. In Nature. Edited by: Nature Publishing Group; 2017:440-445. vol 544.] 
26. Yan L, Wu H, Li X, Gao N, Chen Z: Structures of the ISWI-nucleosome complex reveal a conserved mechanism of chromatin remodeling. In Nature Structural and Molecular Biology. Edited by: Springer US; 2019:258-266. vol 26.]

27. Farnung L, Vos SM, Wigge C, Cramer P: Nucleosome-Chd1 structure and implications for chromatin remodelling. Nature 2017, 550:539-542.

28. Bowman GD, Deindl S: Remodeling the genome with DNA twists. Science 2019, 366:35-36.

29. Eustermann S, Schall K, Kostrewa D, Lakomek K, Strauss M, Moldt M, Hopfner KP: Structural basis for ATPdependent chromatin remodelling by the INO80 complex. In Nature. Edited by; 2018:386-390. vol 556.]

30. Ayala R, Willhoft O, Aramayo RJ, Wilkinson M, McCormack EA, Ocloo L, Wigley DB, Zhang X: Structure and regulation of the human INO80-nucleosome complex. In Nature. Edited by; 2018:391-395. vol 556.]

31. Han Y, Reyes AA, Malik S, He Y: Cryo-EM structure of SWI/SNF complex bound to a nucleosome. Nature 2020, 579:452-455.

32. Wagner FR, Dienemann C, Wang H, Stützer A, Tegunov D, Urlaub H, Cramer P: Structure of SWI/SNF chromatin remodeller RSC bound to a nucleosome. Nature 2020, 579:448-451.

33. Patel AB, Moore CM, Greber BJ, Luo J, Zukin SA, Ranish J, Nogales E: Architecture of the chromatin remodeler RSC and insights into its nucleosome engagement. Elife 2019, 8.

34. Ye Y, Wu H, Chen K, Clapier CR, Verma N, Zhang W, Deng H, Cairns BR, Gao N, Chen Z: Structure of the RSC complex bound to the nucleosome. In Science. Edited by.

35. He S, Wu Z, Tian Y, Yu Z, Yu J, Wang X, Li J, Liu B, Xu Y: Structure of nucleosome-bound human BAF complex. In Science (New York, N.Y.). Edited by; 2020:1-12. vol 9761.]

36. Tosi A, Haas C, Herzog F, Gilmozzi A, Berninghausen O, Ungewickell C, Gerhold CB, Lakomek K, Aebersold R, Beckmann R, et al.: XStructure and subunit topology of the INO80 chromatin remodeler and its nucleosome complex. In Cell. Edited by: Elsevier Inc.; 2013:1207. vol 154.]

37. Mashtalir N, D'Avino AR, Michel BC, Luo J, Pan J, Otto JE, Zullow HJ, McKenzie ZM, Kubiak RL, St Pierre R, et al.: Modular Organization and Assembly of SWI/SNF Family Chromatin Remodeling Complexes. Cell 2018, 175:1272-1288 e1220.

38. Zhou CY, Johnson SL, Gamarra NI, Narlikar GJ: Mechanisms of ATP-Dependent Chromatin Remodeling Motors. In Annual Review of Biophysics. Edited by; 2016:153-181. vol 45.]

39. Willhoft O, Ghoneim M, Lin CL, Chua EYD, Wilkinson M, Chaban Y, Ayala R, McCormack EA, Ocloo L, Rueda DS, et al.: Structure and dynamics of the yeast SWR1-nucleosome complex. Science 2018, 362.

40. Dann GP, Liszczak GP, Bagert JD, Muller MM, Nguyen UTT, Wojcik F, Brown ZZ, Bos J, Panchenko T, Pihl R, et al.: ISWI chromatin remodellers sense nucleosome modifications to determine substrate preference. Nature 2017, 548:607-611.

41. Watanabe $S$, Radman-Livaja M, Rando OJ, Peterson CL: A histone acetylation switch regulates H2A.Z deposition by the SWR-C remodeling enzyme. Science 2013, 340:195-199.

42. Valencia AM, Collings CK, Dao HT, St Pierre R, Cheng YC, Huang J, Sun ZY, Seo HS, Mashtalir N, Comstock DE, et al.: Recurrent SMARCB1 Mutations Reveal a Nucleosome Acidic Patch Interaction Site That Potentiates mSWI/SNF Complex Chromatin Remodeling. Cell 2019, 179:1342-1356 e1323.

43. Racki LR, Yang JG, Naber N, Partensky PD, Acevedo A, Purcell TJ, Cooke R, Cheng Y, Narlikar GJ: The chromatin remodeller ACF acts as a dimeric motor to space nucleosomes. Nature 2009, 462:10161021.

44. Willhoft O, McCormack EA, Aramayo RJ, Bythell-Douglas R, Ocloo L, Zhang X, Wigley DB: Crosstalk within a functional INO80 complex dimer regulates nucleosome sliding. Elife 2017, 6.

45. Grune T, Brzeski J, Eberharter A, Clapier CR, Corona DF, Becker PB, Muller CW: Crystal structure and functional analysis of a nucleosome recognition module of the remodeling factor ISWI. Mol Cell 2003, 12:449-460.

46. Aramayo RJ, Willhoft O, Ayala R, Bythell-Douglas R, Wigley DB, Zhang X: Cryo-EM structures of the human INO80 chromatin-remodeling complex. In Nature Structural and Molecular Biology. Edited by: Springer US; 2018:37-44. vol 25.]

47. Weber J: ATP synthase--the structure of the stator stalk. Trends Biochem Sci 2007, 32:53-56.

48. Schilbach S, Hantsche M, Tegunov D, Dienemann C, Wigge C, Urlaub H, Cramer P: Structures of transcription pre-initiation complex with TFIIH and Mediator. Nature 2017, 551:204-209.

49. Yan L, Wu H, Li X, Gao N, Chen Z: Structures of the ISWI-nucleosome complex reveal a conserved mechanism of chromatin remodeling. Nat Struct Mol Biol 2019, 26:258-266.

50. Li M, Xia X, Tian Y, Jia Q, Liu X, Lu Y, Li M, Li X, Chen Z: Mechanism of DNA translocation underlying chromatin remodelling by Snf2. Nature 2019, 567:409-413. 
51. Widom J: Role of DNA sequence in nucleosome stability and dynamics. Q Rev Biophys 2001, 34:269-324.

52. Langst G, Becker PB: Nucleosome remodeling: one mechanism, many phenomena? Biochim Biophys Acta 2004, 1677:58-63.

53. Brahma S, Udugama MI, Kim J, Hada A, Bhardwaj SK, Hailu SG, Lee TH, Bartholomew B: INO80 exchanges H2A.Z for H2A by translocating on DNA proximal to histone dimers. Nat Commun 2017, 8:15616.

54. Zhou CY, Johnson SL, Lee L, Longhurst AD, Beckwith SL, Johnson MJ, Morrison AJ, Narlikar GJ: The Yeast INO80 Complex Operates as a Tunable DNA Length-Sensitive Switch to Regulate Nucleosome Sliding. In Molecular Cell. Edited by: Elsevier Inc.; 2018:677-688.e679. vol 69.]

55. Myong S, Bruno MM, Pyle AM, Ha T: Spring-loaded mechanism of DNA unwinding by hepatitis C virus NS3 helicase. Science 2007, 317:513-516.

56. Lorch Y, Maier-Davis B, Kornberg RD: Role of DNA sequence in chromatin remodeling and the formation of nucleosome-free regions. Genes Dev 2014, 28:2492-2497.

57. Szerlong H, Hinata K, Viswanathan R, Erdjument-Bromage H, Tempst $P$, Cairns BR: The HSA domain binds nuclear actin-related proteins to regulate chromatin-remodeling ATPases. Nat Struct Mol Biol 2008, 15:469-476.

58. Son EY, Crabtree GR: The role of BAF ( $\mathrm{mSWI} / \mathrm{SNF}$ ) complexes in mammalian neural development. Am J Med Genet C Semin Med Genet 2014, 166C:333-349.

59. Hodges C, Kirkland JG, Crabtree GR: The Many Roles of BAF (mSWI/SNF) and PBAF Complexes in Cancer. Cold Spring Harb Perspect Med 2016, 6.

60. Knoll KR, Eustermann S, Niebauer V, Oberbeckmann E, Stoehr G, Schall K, Tosi A, Schwarz M, Buchfellner A, Korber $\mathrm{P}$, et al.: The nuclear actin-containing Arp8 module is a linker DNA sensor driving INO80 chromatin remodeling. In Nature Structural and Molecular Biology. Edited by: Springer US; 2018:823832. vol 25.]

61. Brahma S, Ngubo M, Paul S, Udugama M, Bartholomew B: The Arp8 and Arp4 module acts as a DNA sensor controlling INO80 chromatin remodeling. Nat Commun 2018, 9:3309.

62. Yen K, Vinayachandran V, Pugh BF: SWR-C and INO80 chromatin remodelers recognize nucleosome-free regions near +1 nucleosomes. Cell 2013, 154:1246-1256.

63. Chandler RL, Brennan J, Schisler JC, Serber D, Patterson C, Magnuson T: ARID1a-DNA interactions are required for promoter occupancy by SWI/SNF. Mol Cell Biol 2013, 33:265-280.

64. Yamada K, Frouws TD, Angst B, Fitzgerald DJ, Deluca C, Schimmele K, Sargent DF, Richmond TJ: Structure and mechanism of the chromatin remodelling factor ISW1a. Nature 2011, 472:448-453.

65. Schubert HL, Wittmeyer J, Kasten MM, Hinata K, Rawling DC, Heroux A, Cairns BR, Hill CP: Structure of an actin-related subcomplex of the SWI/SNF chromatin remodeler. Proc Natl Acad Sci U S A 2013, 110:3345-3350.

66. Turegun B, Kast DJ, Dominguez R: Subunit Rtt102 controls the conformation of the Arp7/9 heterodimer and its interactions with nucleotide and the catalytic subunit of SWI/SNF remodelers. $J$ Biol Chem 2013, 288:35758-35768.

67. Turegun B, Baker RW, Leschziner AE, Dominguez R: Actin-related proteins regulate the RSC chromatin remodeler by weakening intramolecular interactions of the Sth1 ATPase. Commun Biol 2018, 1:1.

68. Ronan JL, Wu W, Crabtree GR: From neural development to cognition: unexpected roles for chromatin. Nat Rev Genet 2013, 14:347-359.

69. Farnung L, Vos SM, Wigge C, Cramer P: Nucleosome-Chd1 structure and implications for chromatin remodelling. In Nature. Edited by: Nature Publishing Group; 2017:539-542. vol 550.] 